\title{
Estimating the magnetic energy inside traveling compression regions
}

\author{
S. A. Kiehas ${ }^{1}$, V. S. Semenov ${ }^{2}$, H. K. Biernat ${ }^{1,3}$, V. V. Ivanova ${ }^{1}$, R. Nakamura ${ }^{1}$, and W. Baumjohann ${ }^{1}$ \\ ${ }^{1}$ Space Research Institute, Austrian Academy of Sciences, Schmiedlstr. 6, 8042 Graz, Austria \\ ${ }^{2}$ Institute of Physics, State University St. Petersburg, 198504 Russia \\ ${ }^{3}$ Institute of Physics, University Graz, Universitätsplatz 5, $8010 \mathrm{Graz}$, Austria
}

Received: 21 October 2008 - Revised: 9 February 2009 - Accepted: 19 February 2009 - Published: 4 May 2009

\begin{abstract}
We investigate a series of six TCRs (traveling compression regions), appearing in the course of a small substorm on 19 September 2001. Except for two of these TCRs, all Cluster spacecraft were located in the lobe and detected the typical signatures of TCRs, i.e., compressions in $|B|$ and bipolar $B_{z}$ variations. We use these perturbations in $B_{z}$ for calculations on the magnetic energy inside the TCR and compare the amount of magnetic field energy with the kinetic energy inside the underlying plasma bulge. According to results obtained from theory, the amount of magnetic energy inside TCRs is about two times higher than the kinetic plasma energy inside the accompanied plasma bulge. We verify this theoretical result by first investigations of the magnetic field energy inside TCRs. The calculations lead to a magnetic energy in the order of $10^{10}$ Joule per $R_{E}$ for each of the TCRs.
\end{abstract}

Keywords. Magnetospheric physics (Magnetotail; Storms and substorms) - Space plasma physics (Transport processes)

\section{Introduction}

Within the Near-Earth Neutral Line (NENL) model of substorms (Baker et al., 1996), substorm-associated magnetotail reconnection evolves and leads to several transient and localized mesoscale phenomena with typical temporal scales of several minutes and spatial scales of a few Earth radii (Sharma et al., 2008). One of these phenomena are traveling compression regions (TCRs, Slavin et al., 1984).

TCRs can be seen as regions of locally and temporally enhanced magnetic field magnitude, due to a transient thickening of the plasma sheet, i.e., a plasma bulge, traveling along

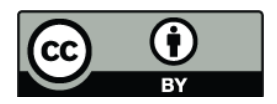

Correspondence to: S. A. Kiehas (stefan.kiehas@oeaw.ac.at) the current sheet in the Earth's magnetotail and compressing the magnetic field lines above and beneath it. Since this plasma bulge is propagating along the current sheet, the corresponding field compression is traveling as well.

First indications for the appearance of TCRs, the characteristic reversal in $B_{z}$ associated with a smooth magnetic field compression, were found during Explorer 35 observations in the 1970s (Maezawa, 1975). TCRs were investigated in more detail in the distant tail, $X<-30 R_{E}$, by ISEE-3 observations with a frequency of several events per day (Slavin et al., 1984). These distant TCRs last for several minutes and exhibit a magnetic field compression of $\Delta B / B \sim 5-10 \%$ in $|B|$, associated with a north-to-south (NS) reversal of $B_{z}$ with the inflection near the time of peak compression in $|B|$ (Slavin et al., 1984). Due to the magnetic field configuration of the Earth's magnetotail, a NS reversal in $B_{z}$ indicates a propagation of the TCR down the tail. Early it was suggested that TCRs are the lobe signatures of plasmoids/flux tubes, which locally increase the plasma sheet thickness and thus, compress the surrounding lobe regions, i.e., the lobe's magnetic field. These plasmoids were described as large magnetic islands, resulting from magnetotail reconnection, and travel rapidly down the tail during substorms (e.g., Hones Jr. et al., 1984). The relation to substorms is also shown in Slavin et al. (1993), where it was found that over $91 \%$ of all investigated TCRs followed substorm onsets or intensifications.

During their study of earthward propagating TCRs, Slavin et al. (2005) showed that these TCRs tend to be observed during the expansion phase of substorms by superposed epoche analysis of the Kyoto World Data Center Quick Look AL Index, compared with the time of the TCRs in 2001. Extrinsic influences, like a compression of the entire magnetosphere by interplanetary shock waves or compressive tail oscillation modes excited by the flow of the solar wind around the tail, can be ruled out for several reasons, as pointed out by Slavin et al. (1984). Besides extensive studies of TCRs in the distant

Published by Copernicus Publications on behalf of the European Geosciences Union. 

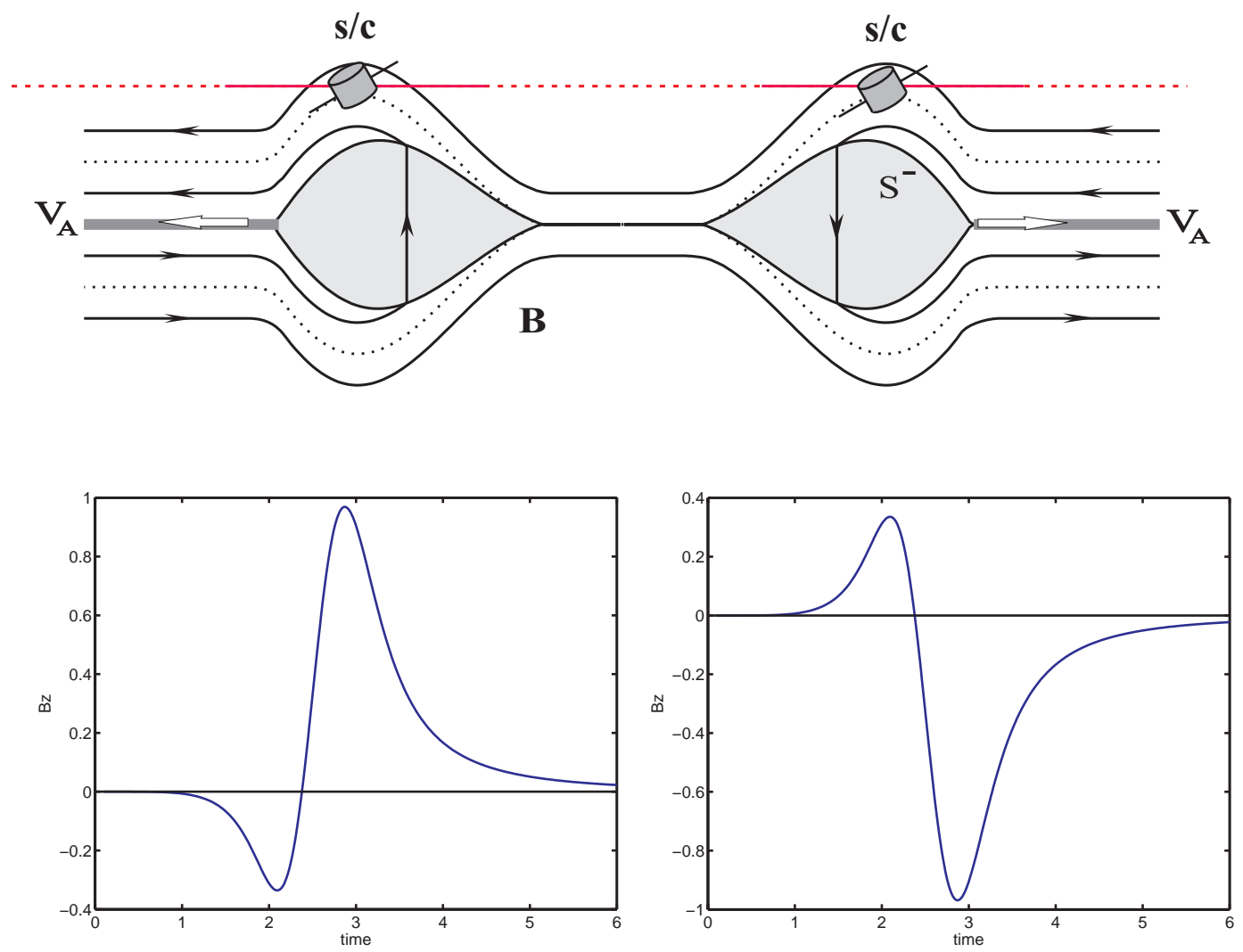

Fig. 1. Disturbances in $B_{z}$ measured by spacecraft on opposite sides of the reconnection line. The leftwards positioned spacecraft measures first negative and then positive $B_{z}$ disturbances, when the plasma outflow region passes by. For the rightward positioned spacecraft the situation is vice versa. The field line configuration corresponds to those of the Earth's magnetotail.

tail by ISEE-3 (e.g., Moldwin and Hughes, 1993; Slavin et al., 1993) and Geotail (Shirai et al., 2001), TCRs were also investigated in the mid-tail between $-25 R_{E}>X>-38 R_{E}$ by IMP 8 (Slavin et al., 1990; Moldwin and Hughes, 1994). A thorough analysis of TCRs observed during the ISEE-3 magnetic field observations can be found in Slavin et al. (1993), where 116 TCRs, appearing as isolated, paired, or multiple events, are used as a basis for a statistical study. The mean compression of these tailward propagating TCRs was $\Delta B / B=7.6 \%$ with a duration of $158 \mathrm{~s}$. The size of these plasmoids was estimated by the TCR amplitude and duration to be approximately $35 R_{E}$ in x- and $15 R_{E}$ in y- and z-direction. IMP 8 observations in the mid-tail gave first evidence of the existence of earthward propagating TCRs (Moldwin and Hughes, 1994; Moldwin et al., 2001), which was supported by Cluster observations in the near tail at $X>-20 R_{E}$. These near tail TCRs (Slavin et al., 2003c; Borälv et al., 2005; Sergeev et al., 2005; Owen et al., 2005) strongly resemble those in the distant tail. Nevertheless, several differences can be found between the near tail and distant tail TCRs, as a comprehensive analysis of 148 TCRs, observed during the 2001-2002 Cluster tail season and discussed in Slavin et al. (2005), shows:
1. $80 \%$ of the observed near tail TCRs exhibt a south-tonorth turning in $B_{z}$, indicating an earthward propagation. 20\% show a north-to-south reversal, i.e., a tailward propagation, as it appears for all observed distant tail TCRs.

2. The mean duration of the compression is just $\sim 35 \mathrm{~s}$ in contradiction to about $160 \mathrm{~s}$ lasting TCRs in the distant tail at $X<-60 R_{E}$.

3. The mean width of the 148 TCRs evaluated in Slavin et al. (2005) was found to be $4.3 R_{E}$. Distant tail plasmoids exhibit an elongation of $35 R_{E}$ in $x$ and $15 R_{E}$ in $z$.

Geotail observations indicate the appearance of small flux ropes with a diameter of a few Earth radii in the near tail ( $X>-30 R_{E}$, Slavin et al., 2003a,b), which are immersed within earthward propagating BBFs. Therefore, Slavin et al. (2003c) proposed to distinguish between mainly earthward propagating brief TCRs in the near tail and tailward propagating long lasting TCRs by different types of underlying flux ropes, i.e., "BBF-type" and "plasmoid-type" flux ropes, respectively. 


\section{The substorm event on 19 September 2001}

Borälv et al. (2005) reported and described a small substorm, taking place on 19 September 2001. The Kilpisjärvi ASC (All Sky Camera) system observed an intensification of the auroral brightening between 20:38:40 and 20:39:00 UT and ground-based magnetograms detected a decrease in the $B_{x}$ component of the magnetic field with a negative bay reaching about $-100 \mathrm{nT}$, indicating the appearance of a weak substorm starting at 20:39 UT. The appearance of hightemperature electrons and an increase of the electron density at lower altitudes of precipitating electrons, confirm the beginning of the expansion phase at 20:39 UT. The start of the recovery phase can be found at about 22:15 UT by a gradual decay of $B_{x}$ to the baseline before the substorm onset. Due to a lack of geosynchronous observations of energetic particle injections, this substorm can be considered as small.

At 20:39 UT the barycenter of the Cluster tetrahedron was located at about $(X, Y, Z)=(-18.6,5.3,0.35) \mathrm{GSE}$; duskwards in the Southern Hemisphere. As can be seen from Fig. 2, the Cluster spacecraft were located inside the plasma sheet before the onset. According to the energy flux spectrograms, the Cluster s/c drop out the plasma sheet shortly after the ground-based noticed onset of the substorm at 20:39 UT, marked by the first vertical line. The increase in the energy flux corresponds to short excursions of the Cluster s/c into the plasma sheet or PSBL.

At about 20:57 UT, 17 min after substorm onset, Cluster located in the lobe - observed an enhancement in $|B|$ and a bipolar variation in $B_{z}$, indicating the appearance of a TCR, followed by five more TCRs. These TCRs might be produced by underlying BBF-type flux ropes, which travel as bulges of the plasma sheet. The short-time visits of Cluster s/c 1, 2 and 4 of the plasma sheet/PSBL during two TCRs, associated with high-velocity, $600-800 \mathrm{~km} / \mathrm{s}$, earthward directed beams, detected by s/c 1 and 4 (Borälv et al., 2005) can be interpreted as swap over of the plasma bulge over these s/c.

\section{Substorm associated TCRs}

We analyze the six TCRs, appearing between 20:55 UT and 21:57 UT in more detail. Table 1 gives an overview of these TCRs, with respect to the direction of their reversal, time of appearance and velocity of the TCRs.

\subsection{TCR1}

At about 20:55:54 UT, a significant decrease in the $B_{z}$ component measured onboard all Cluster spacecraft can be observed. At 20:57:14 UT, an intensification of the total magnetic field strength of about 1 to $1.5 \mathrm{nT}$ appears, accompanied by a reversal of the magnetic field $B_{z}$ component from negative to positive values at about 20:57:10 UT (see Fig. 3). The maximum increase in the total magnetic field strength as well as the maximum in the $B_{x}$ disturbance appear around the $B_{z}$

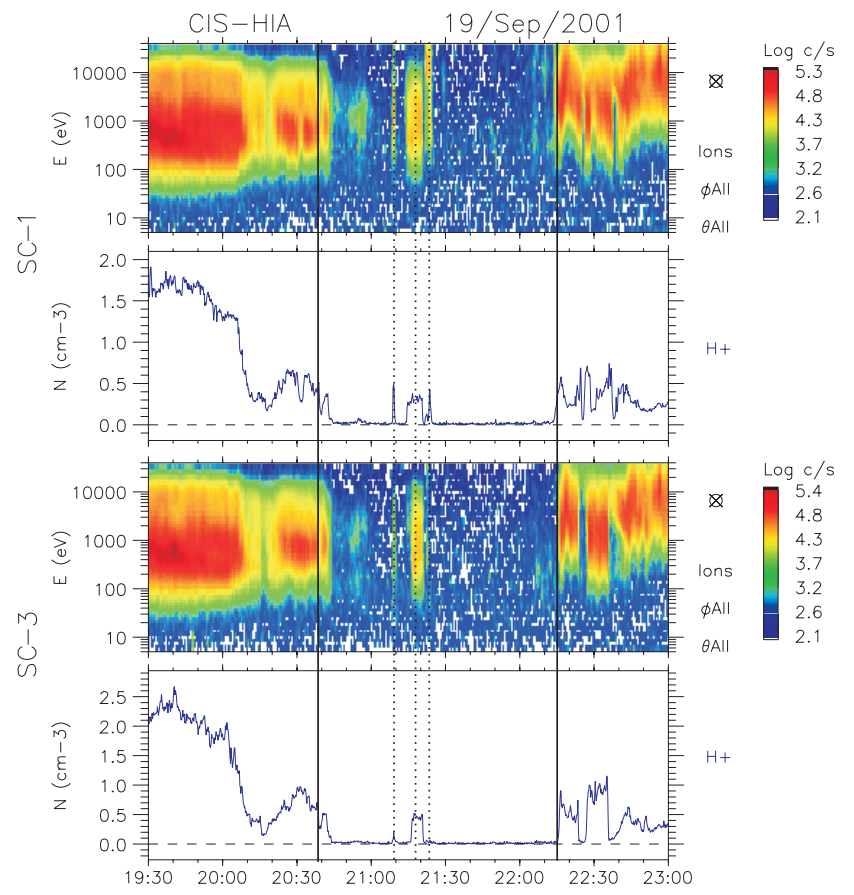

Fig. 2. $\mathrm{H}^{+}$ion densities and energy flux spectrograms from $\mathrm{C} 1$ and C3. As can be seen, the spacecraft are located inside the plasma sheet until the substorm onset at about 20:39 UT (first solid vertical line). Before substorm onset, Cluster detected high-energy ions and a high particle density. During the substorm, i.e., until the beginning of the recovery phase at about 22:15 UT (second solid vertical line), no high-energy ions were detected and the particle density drops down, indicating Cluster being in the lobe. Several intensifications of the energy flux and enhancements of the plasma density are due to short excursions back into the plasma sheet or PSBL. As can be seen, Cluster s/c 3 remains in the lobe for the first and third excursion of s/c 1 (dotted lines).

reversal. The south-then-north reversal of $B_{z}$ indicates that this TCR travels earthwards, which is confirmed by TOF calculations for the velocity of the TCR (Slavin et al., 2003c).

\subsection{TCR2}

After a quiet period of about nine minutes, all spacecraft observe a decrease in $B_{z}$ at about 21:08:22 UT, depicting the start of a bipolar $B_{z}$ variation until about 21:09:46 UT, with a south-then-north reversal at 21:08:54 UT for C3. This TCR represents a special situation. As can be seen from Fig. 4, observations from $\mathrm{C} 1, \mathrm{C} 2$ and $\mathrm{C} 4$ are significantly different to the observations from $\mathrm{C} 3$. All observations give rise to the assumption that $\mathrm{C} 1, \mathrm{C} 2$ and $\mathrm{C} 4$ are inside the plasma bulge whereas $\mathrm{C} 3$ stays in the lobe. For C3, the typical $B_{x}$ behavior for observing a TCR associated with an earthward flow in the southern lobe-hemisphere can be seen. Firstly, $B_{x}$ increases to about $32 \mathrm{nT}$, and finally decreases to $29 \mathrm{nT}$. $\mathrm{C} 1, \mathrm{C} 2$ and $\mathrm{C} 4$ detect a strong decrease to about $24 \mathrm{nT}$. This 
Table 1. Overview of the TCRs on 19 September 2001. The reversal time corresponds to the change of sign in $B_{z}$. The time of appearance displays the time interval between the onset and the offset of the disturbance in $B_{z}$. The speed of the TCR was determined by Slavin et al. (2003c) via time of flight (TOF) calculations by using lag correlations in magnetic field measurements between s/c 1 and 4 , which had the largest separation. Therefore, the difference in the pass-by time of the TCRs at s/c 1 and 4 can been taken to determine the speed of the TCRs. Except C3, all Cluster s/c were swept over by the plasma bulge during the second and third TCR.

\begin{tabular}{lcccccc}
\hline & TCR1 & TCR2 & TCR3 & TCR4 & TCR5 & TCR6 \\
\hline$B_{z}$ reversal & $20: 57: 10$ & $21: 08: 54$ & $21: 23: 34$ & $21: 33: 50$ & $21: 39: 46$ & 21:55:10 \\
time (UT) & & & & & & \\
Time of & $20: 55: 54$ & $21: 08: 22$ & $21: 22: 46$ & $21: 32: 30$ & $21: 36: 26$ & 21:53:06 \\
appearance (UT) & $20: 59: 22$ & $21: 09: 46$ & $21: 24: 46$ & $21: 35: 58$ & $21: 43: 38$ & $21: 56: 54$ \\
Reversal & South & South & South & South & North & South \\
direction & North & North & North & North & South & North \\
TCR speed & 415 & 416 & 594 & 377 & -259 & 415 \\
$\begin{array}{l}\text { Slavin et al., 2003c) } \\
\text { Cluster }\end{array}$ & Lobe & bulge & bulge & Lobe & Lobe & Lobe \\
position & & (exc. C3) & (exc. C3) & & & \\
\hline
\end{tabular}

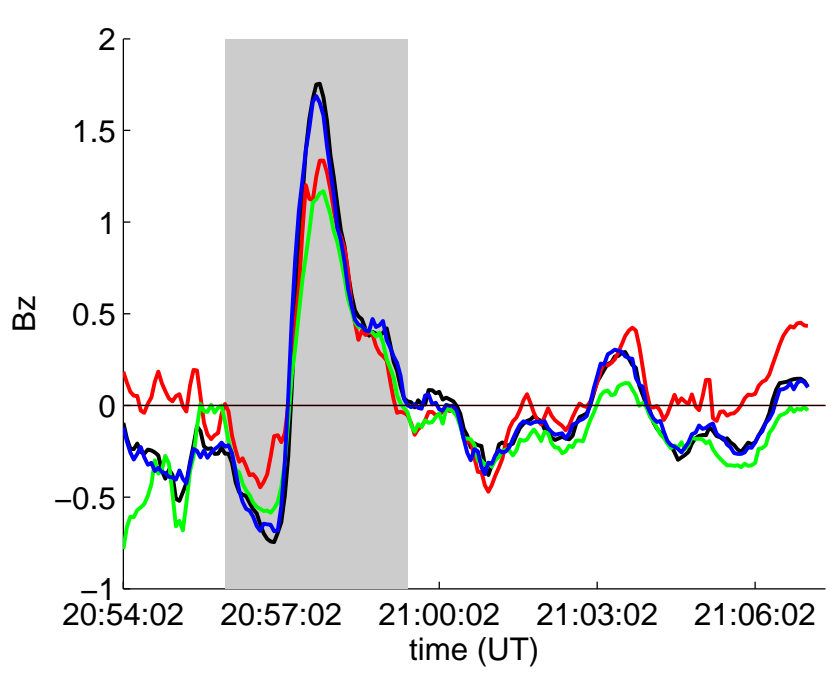

Fig. 3. TCR1: Detrended $B_{z}$ data observed by Cluster spacecraft during 20:54:02 and 21:07:02 UT. The typical bipolar signature is visible for all spacecraft.

supports our picture of the $B_{x}$ behavior inside a plasma outflow region, where $B_{z}$ is increased and $B_{x}$ decreased, as the sketch in Fig. 5 displays. Whereas $\mathrm{C} 1, \mathrm{C} 2$, and $\mathrm{C} 4$ are located between $z=-1.03$ and $z=-1.08 R_{E \text { GSM }}, \mathrm{C} 3$ is positioned at $z=-1.3 R_{E \text { GSM }}$. The situation can be described by analyzing the plasma density data, shown in Fig. 6. During the second TCR at 21:08:54 UT, the plasma density, detected by $\mathrm{C} 1$ and $\mathrm{C} 4$ is highly increased, indicating that the plasma flow, associated with the TCR, swaps over these spacecraft. $\mathrm{C} 3$, on the other hand, detects a relatively small enhancement of the plasma density, which leads to the assumption that $\mathrm{C} 3$ is still outside the plasma sheet. Therefore, the distance between $\mathrm{C} 3$ and the other spacecraft can be seen as z-distance of the outflow region.

\subsection{TCR3}

After a rather turbulent time, the next TCR could be observed with an onset at 21:22:46 UT. This event depicts a good example for the importance of $B_{x}$ to identify a TCR, shown in Fig. 7. Due to the strong fluctuations between 0 and $4 \mathrm{nT}$ in $B_{z}$, a TCR-related variation is hard to identify by using only $B_{z}$ data. Beside variations in $B_{z}$, a TCR appears along with an increase in the magnitude of $\left|B_{x}\right|$, observed at 21:23:34 UT. This intensification in $B_{x}$ is correlated with a TCR-associated variation in $B_{z}$. This variation lasts till 21:24:46 UT. Similar to the second TCR, the measured magnetic field from $\mathrm{C} 1, \mathrm{C} 2$ and $\mathrm{C} 4$ differ from that from $\mathrm{C} 3$, leading to the same conclusion as for TCR2: Only C3 is located in the lobe, whereas the other $\mathrm{s} / \mathrm{c}$ are swaped over by the plasma flow. This picture is supported by the plasma data in Fig. 6.

\subsection{TCR4, TCR5, TCR6}

During the time 21:32:30 and 21:35:58 UT, the appearance of the fourth TCR, it can be seen that all spacecraft measure a similar behavior of the magnetic field, indicating that the entire Cluster tetrahedron is located inside the lobe region.

Only about $80 \mathrm{~s}$ after the pass-by of the fourth TCR, a new disturbance in $B_{z}$ is detected by all four Cluster s/c. In contradiction to the first four TCRs, $B_{z}$ displays a north-thensouth reversal, indicating the detection of a tailward propagating structure. This assumption gets affirmed by TOF analysis of the TCR velocity by Slavin et al. (2003c). The velocity was measured to be $-259 \mathrm{~km} / \mathrm{s}$, and thus, the TCR travels towards the tail. This leads to the assumption of a reconnection line evolved somewhere in the region $X<-18 R_{E}$. We identify the disturbance to start at about 21:36:26 UT with a reversal at about 21:39:46UT. Although the southward disturbance in $B_{z}$ recovers at about 21:41:38 UT, this should not 

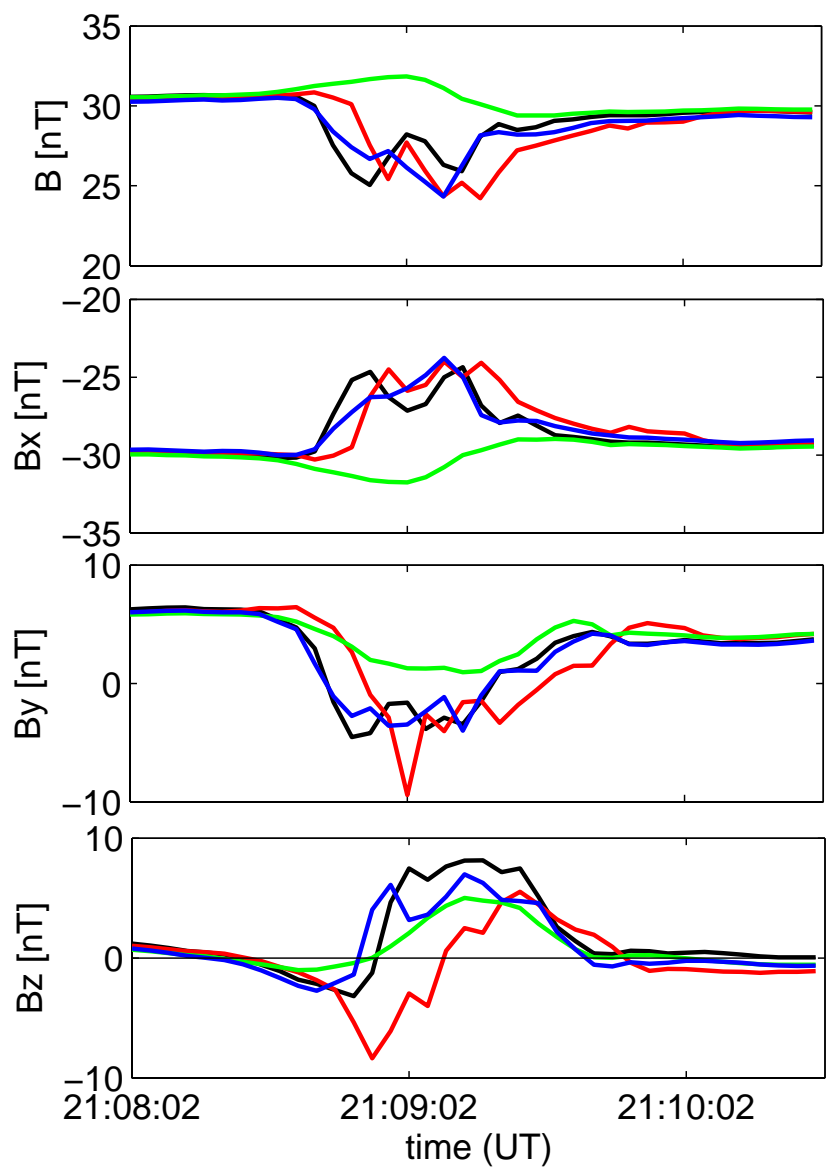

Fig. 4. TCR2: Total magnetic field $B$, and $B_{x}, B_{y}$, and $B_{z}$ for the second TCR. The different behavior of C1 (black), C2 (red), C4 (blue) data on the one hand and $\mathrm{C} 3$ (green) data on the other hand is due to the location of the first mentioned spacecraft inside the plasma bulge and $\mathrm{C} 3$ outside.

be seen as the end of the variation. Figure 8 shows that the $B_{x}$ disturbance lasts until about 21:43:38 UT. This corresponds to a second recovering of $B_{z}$. Thus, it is more reasonable to identify this second recovering of $B_{z}$ at 21:43:38 UT as the end of the TCR-related variation. A second argument for this perception is the fact that the southward period until the first recovering is shorter and exhibits a smaller magnitude than the northward period before the reversal. This leads to the transport of an unphysical negative amount of reconnected flux. We assume that the appearance of two minima in $B_{z}$ is due to external influences which disturb the TCR-related variation in $B_{z}$. Since we do neither know the shape nor the magnitude of the TCR-related variation, we exclude TCR5 from our investigations.

The appearance of the last TCR of this series of events is noticed at about 21:53:06 UT by the typical variations in the magnetic field observations, shown in Fig. 9. The data from $\mathrm{C} 2$ should be omitted for this investigation, since the

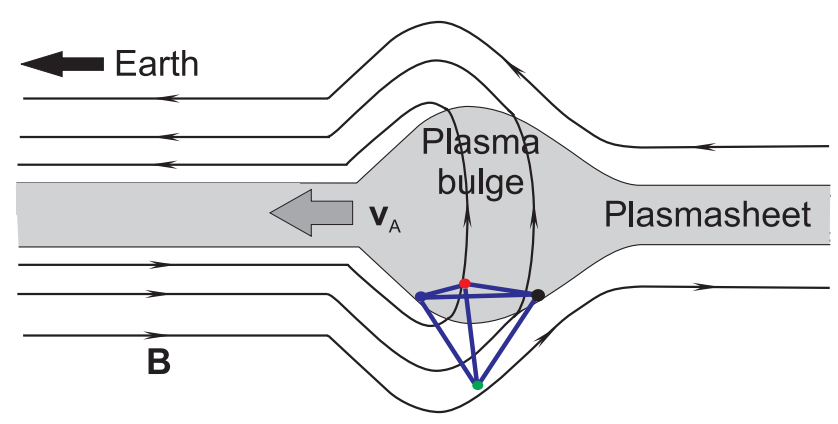

Fig. 5. Sketch for the situation as it appears during TCR 2 and TCR3. The plasma bulge swaps over s/c C1, C2 and C4, whereas $\mathrm{C} 3$ remains outside the plasma sheet in the lobe region. Due to the different magnetic field lines configuration, the spacecraft inside the plasma bulge detect a decrease in $B_{x}$ and in the total magnetic field strength as well as an increase in $B_{z}$.

magnetic field measurements of $\mathrm{C} 2$ from Fig. 10 give raise to the assumption that $\mathrm{C} 2$ shortly enters a flux rope at the beginning of the disturbance, indicated by a strong increase in $B_{y}$.

\section{The kinetic energy inside the outflow region}

The kinetic energy of the plasma inside the outflow region can be calculated via

$W_{k}=\int \frac{\rho v^{2}}{2} d V$

where $\rho, v$ and $V$ denote the plasma density, plasma velocity and volume of the outflow region, respectively. With a typical particle density inside the plasma sheet of $n=0.5 \mathrm{~cm}^{-3}$, and the hydrogen ions as main contributors to the plasma sheet population, the density $\rho$ can be found to be $\rho=8.5 \times 10^{-25} \mathrm{~g} / \mathrm{cm}^{3}$, via $\rho=n m_{p}$, where $m_{p}=1.7 \times 10^{-24} \mathrm{~g}$ is the proton mass. The velocity of the TCR can be seen as average bulk flow velocity of the plasma in the underlying outflow region, since the TCR propagates together with the plasma bulge. Hence, we use the values of the TCR speed obtained via TOF analysis in Slavin et al. (2003c) as bulk flow velocity of the plasma for the calculations of $W_{k}$.

The shape of the outflow region is approximated as that of an ellipse, which gives an area of

$A=\pi a b$,

with $a$ and $b$ as semi-major and semi-minor axis, corresponding to the half $\mathrm{x}$ - and half $\mathrm{z}$-elongation of the outflow region, respectively. For calculating the kinetic energy, we use half of this area, since we compare the kinetic and magnetic energies for one lobe-hemisphere. According to Slavin et al. (2003c), the half z-elongation can be approximated for the second and third TCR as $0.5 R_{E}$, since $\mathrm{C} 1, \mathrm{C} 2$, and $\mathrm{C} 4$ find 


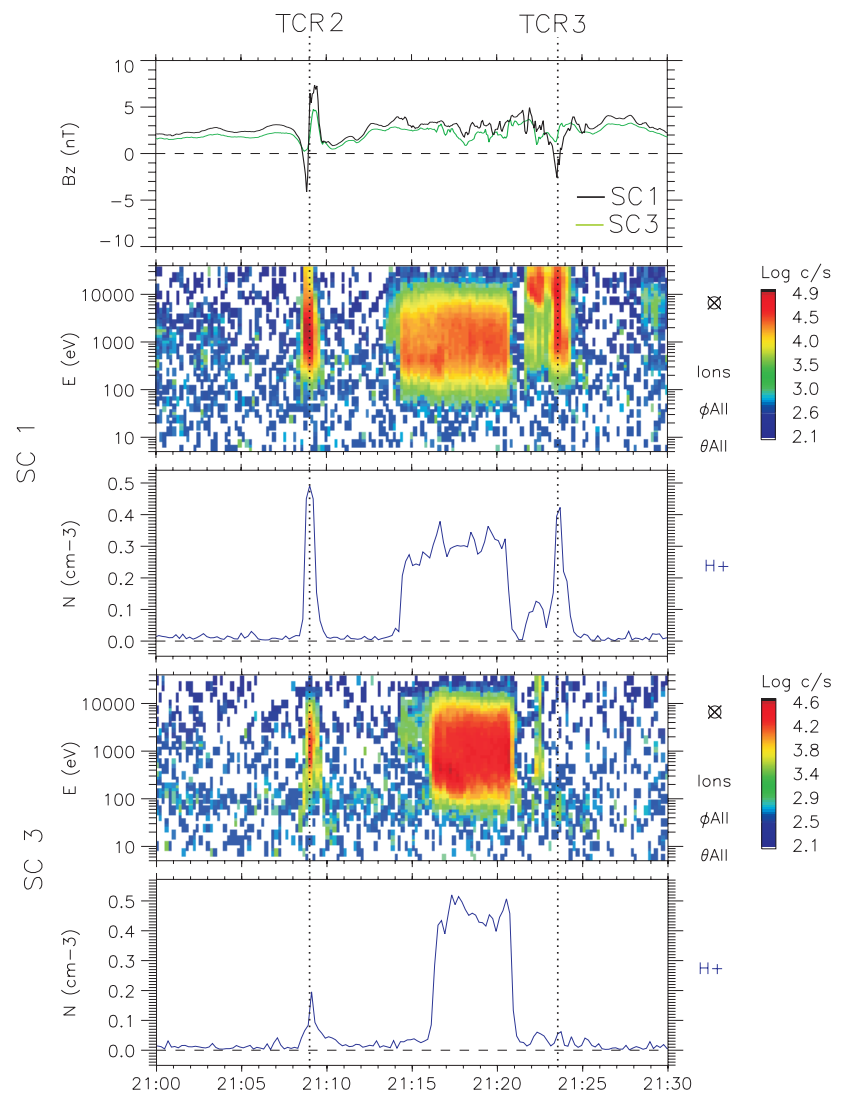

Fig. 6. Cluster magnetic field and plasma observations during the second (21:08:54 UT) and third (21:23:34 UT) TCR. An enhancement of the plasma density for $\mathrm{C} 1$ can be seen during the second and third TCR (C2 and C4 show similar behavior as C1, see Slavin et al. (2003c) for details), whereas C3 detects only a small increase.

themselves inside the plasma bulge for these TCRs, whereas $\mathrm{C} 3$ stays outside. Therefore, the distance between $\mathrm{C} 3$ and the surface, spanned by the other spacecraft, can be seen as approximated maximum elongation of the outflow region. We assume a similar z-elongation for the other TCRs as well.

When reconnection starts, the outflow region begins to evolve and expands in x-direction. After the time $T$, the duration of the reconnection pulse, the reconnection electric field drops to zero and the expansion of the outflow region stops. It detaches from the initial reconnection site and propagates with local Alfvén velocity and constant $\mathrm{x}$-elongation along the current sheet. Therefore, the extension in $x$ corresponds to $x=v_{A} T$. We assume the duration $T$ of the reconnection pulse to be $50 \mathrm{~s}$, which is found to be a typical value (e.g., Semenov et al., 2005). With $b=0.5 R_{E}$ and $a=v_{A} T / 2=1.63 R_{E}$, we find the area of the outflow region in $X-Z$, to be $2.55 R_{E}^{2}$. The calculations are based upon our two-dimensional reconnection model. Therefore, we take $1 R_{E}$ as reference length. Thus, the volume of the outflow region is approximated to be $V=2.55 R_{E}{ }^{3}$. It has to be men-

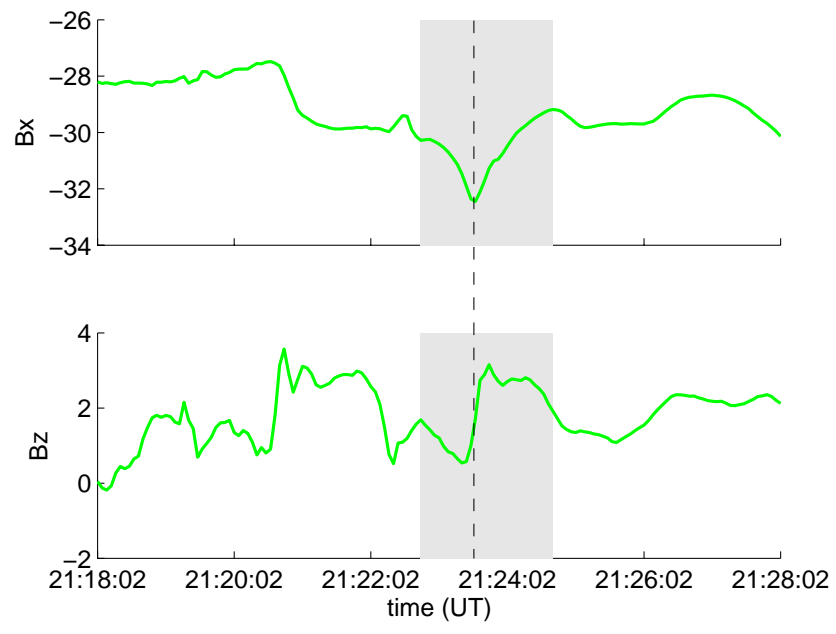

Fig. 7. TCR $3: B_{x}$ and $B_{z}$ observed by $\mathrm{C} 3$ during a period with quite strong fluctuations in $B_{z}$. The increase in the value of $\left|B_{x}\right|$ at 21:23:34 UT indicates a TCR and is consistent with the variation in $B_{z}$.

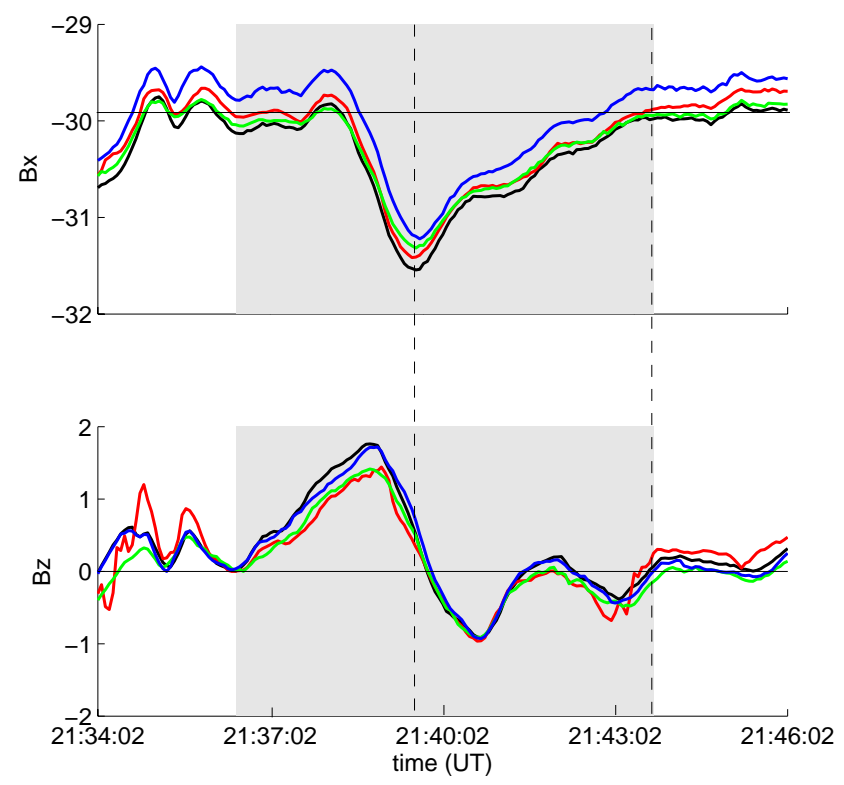

Fig. 8. TCR5: $B_{x}$ and shifted $B_{z}$ for the fifth TCR. The horizontal line in the upper panel denotes the approximated background $B_{x}$ with respect to $\mathrm{C} 1, \mathrm{C} 2$ and $\mathrm{C} 3$. The variation in $B_{x}$ ends at about 21:43:38 UT, denoted by a vertical dashed line. The first dashed line represents the maximum in $\left|B_{x}\right|$.

tioned that the velocity of the outflow region in the magnetotail does not correspond exactly to the local Alfvén speed, as it results from the theoretical model. Hence, we use for the calculations not the local Alfvén velocity but the plasma bulk flow velocity as obtained from TOF analysis, which corresponds to the velocity of the outflow region. 
Table 2. Kinetic plasma energy $W_{k}$ in Joule per unit length of the reconnection line for the outflow regions associated with the investigated TCRs.

\begin{tabular}{cccccc}
\hline & TCR1 & TCR2 & TCR3 & TCR4 & TCR6 \\
\hline$W_{k}$ & $2.4 \times 10^{10}$ & $2.4 \times 10^{10}$ & $7.1 \times 10^{10}$ & $1.8 \times 10^{10}$ & $2.4 \times 10^{10}$ \\
\hline
\end{tabular}
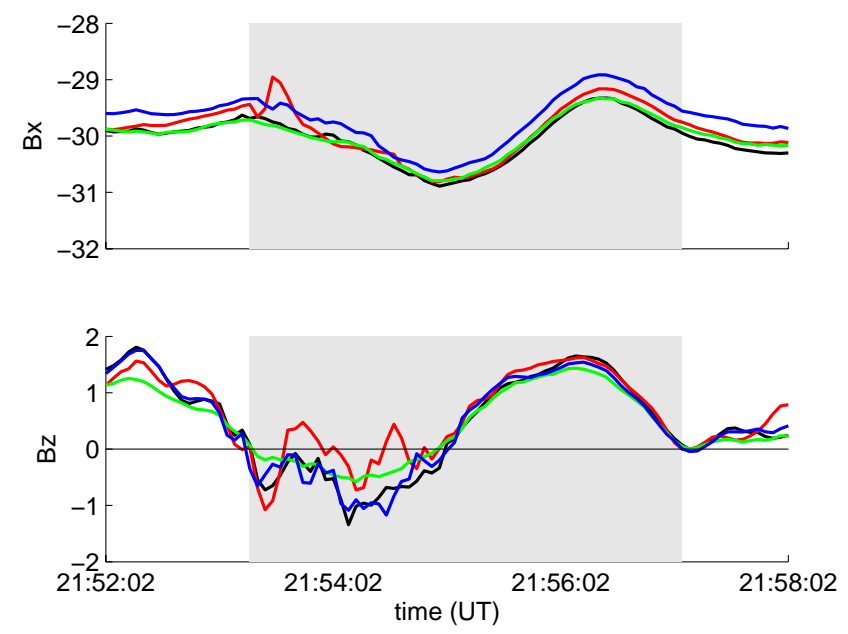

Fig. 9. TCR6: $B_{x}$ data (upper panel) and detrended and for +0.45 shifted $B_{z}$ data (lower panel) for the disturbance appearing between 21:53:06 and 21:56:54 UT.

\section{The magnetic energy inside the compression region}

Due to the appearance of the plasma outflow region, the magnetic field in the region above the outflow regions gets disturbed, i.e., the magnetic field density in this region is enhanced. With the initial background magnetic field exhibiting only an $\mathrm{x}$-component, the magnetic field in the compression regions $B_{1}$ exhibits in a 2-D configuration a component in $x$ in the form $B_{x}=B_{0}+B_{x}^{(1)}$ and in $z, B_{z}=B_{z}^{(1)}$, where $B_{0}$ corresponds to the background magnetic field and $B_{x, z}^{(1)}$ to disturbances in the magnetic field due to the compression region. In order to compare observational results with theoretical ones, the 2-D configuration from Semenov et al. (1998) and Kiehas et al. (2009) is used. The additional magnetic field energy (all equations are written in cgs-units) in the compression region appears as

$\Delta W_{B}=\int\left(\frac{B_{1}^{2}}{8 \pi}-\frac{B_{0}^{2}}{8 \pi}\right) d V$

This gives after neglecting terms of the second order,

$\Delta W_{B}=\frac{B_{0}}{4 \pi} \iint B_{x}^{(1)} d x d z$
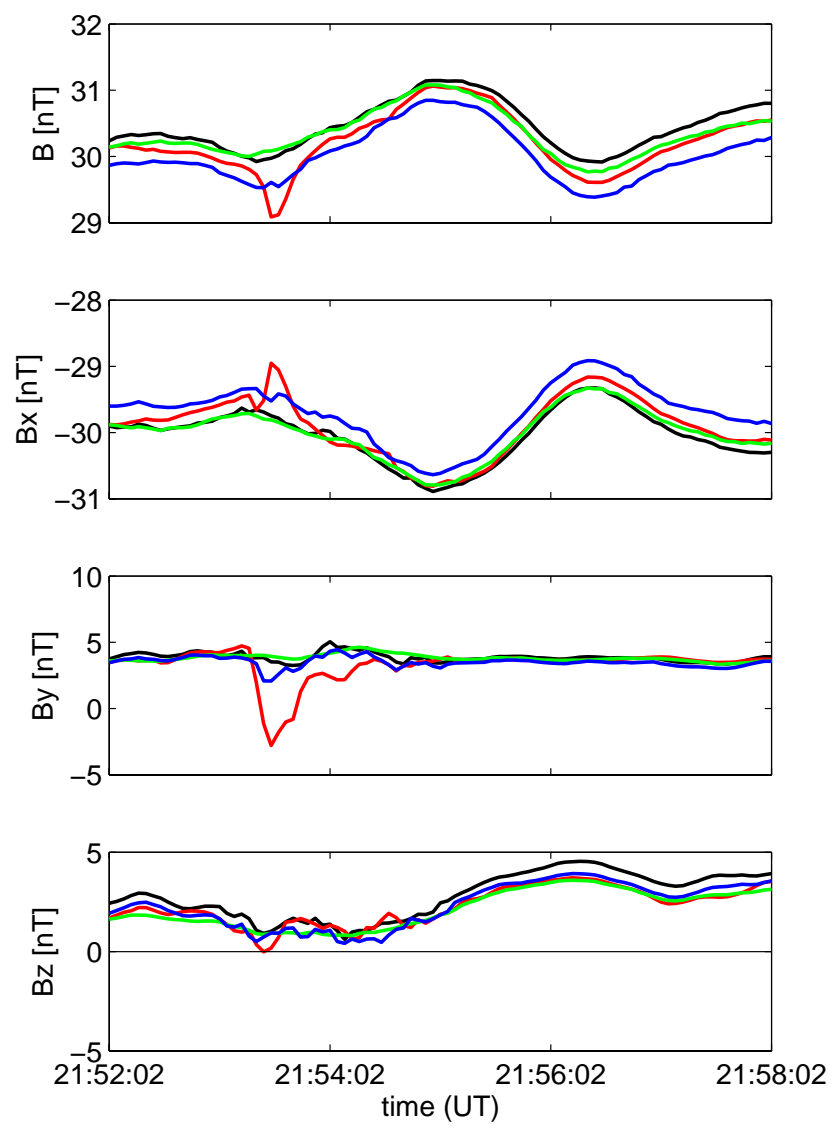

Fig. 10. TCR6: Total magnetic field $B$ and magnetic fields $B_{x}, B_{y}$, and $B_{z}$ for the sixth TCR.

With the vector potential $\mathbf{A}=(0, A, 0)$ of the form $\mathbf{B}=\nabla \times \mathbf{A}$, $B_{x}^{(1)}$ can be expressed in terms of $A$,

$B_{x}^{(1)}=-\frac{\partial A}{\partial z}$.

With this, the magnetic energy can be displayed by the integral over a vector potential,

$\Delta W_{B}=-\frac{B_{0}}{4 \pi} \int_{0}^{\infty} \int_{0}^{\infty} \frac{\partial A}{\partial z} d x d z$

Since the magnetic potential $\mathbf{A}$ is defined to be zero at infinity, this leads with $d x=v_{A} d t$ to,

$\Delta W_{B}=\left.\frac{v_{A} B_{0}}{4 \pi} \int_{0}^{\infty} A\right|_{z=0} d t$. 

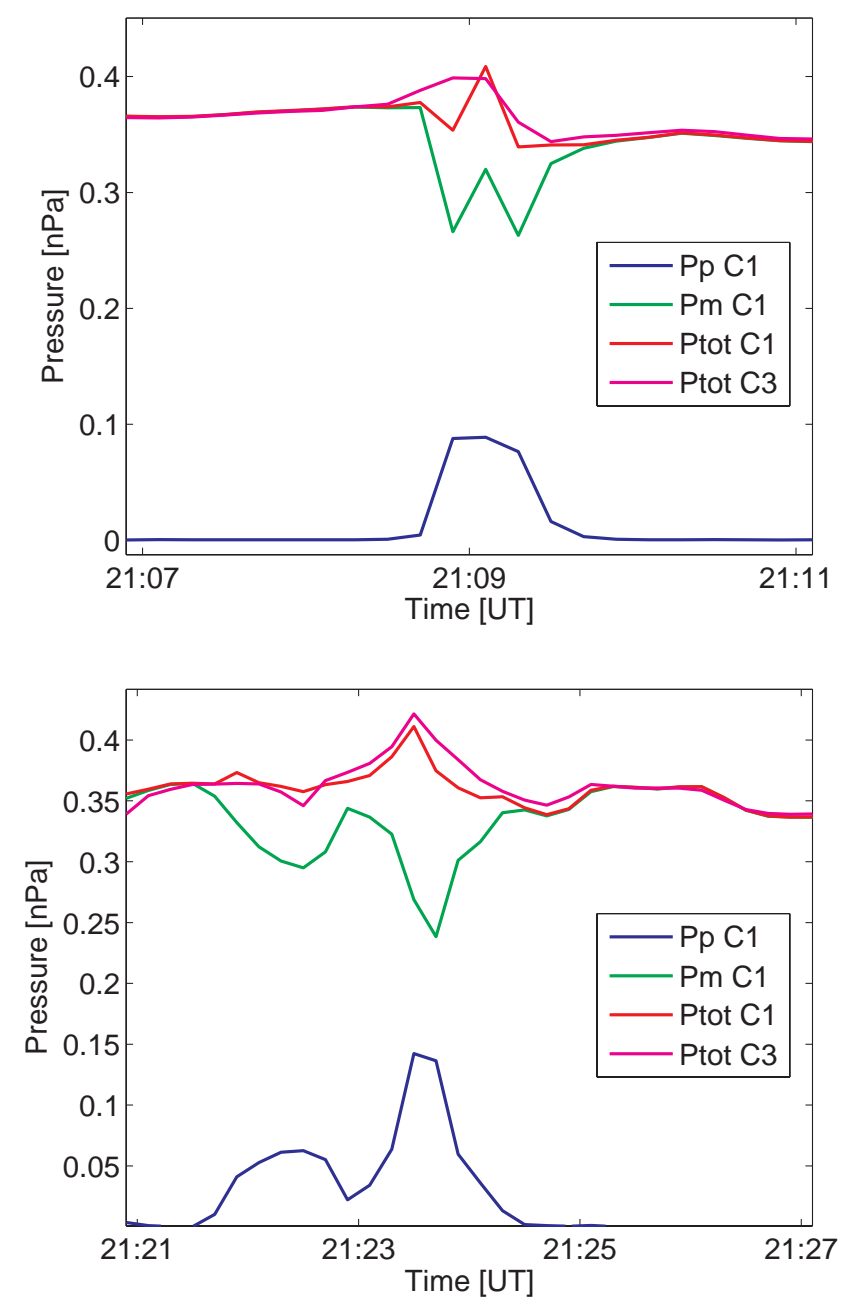

Fig. 11. During TCR2 (upper panel) and TCR3 (lower panel) spacecraft $\mathrm{C} 1$ was located inside the plasma flow and $\mathrm{C} 3$ outside the flow. The total pressure inside the outflow region (red), composed of the plasma pressure (blue) and the magnetic pressure (green) corresponds well with the total pressure outside the outflow region (magenta).

With the relation $B_{z}^{(1)}=\frac{\partial A}{\partial x}$ and $d x=v_{A} d t$ it follows,

$\left.A\right|_{z=0}=\left.v_{A} \int_{0}^{\infty} B_{z}^{(1)}\right|_{z=0} d t$.

Since the disturbance in $B_{z}$ enters this equation as boundary condition, we can determine the amount of magnetic energy inside the compression region from Eqs. (4) and (5), as long as the spacecraft is sufficiently close to the outflow region in z-direction. The velocity $v_{A}$ is taken from Slavin et al. (2003c), who obtained the velocities of the TCRs from a TOF analysis (see also Table 1). Since the TCRs propagate together with the underlying plasma bulge, the speed of the TCRs corresponds to the bulk velocity of the plasma in the associated plasma bulge, i.e., to $v_{A}$ in Eqs. (4) and (5). The results are summarized in Table 3 . The last column shows the ratio of magnetic field energy inside the TCR to the kinetic plasma energy inside the plasma outflow regions. Theoretical calculations suggest a value of about 2 (Semenov et al., 1998; Kiehas et al., 2009) For this first observational investigations, the ratio is about 2.4 for TCR1, TCR4, and TCR6. For TCR2 and TCR3 we could only use C3, since all other spacecraft engulfed the outflow region, and the results deviate more from the theoretical value.

\section{Pressure balance across the boundary}

Due to the location of the Cluster spacecraft during the passage of TCR2 and TCR3 (i.e., C3 observing a TCR, whereas all other spacecraft being inside the plasma flow), one can compare the total pressure inside the plasma outflow region with the total pressure outside. Figure 11 shows the total pressure from $\mathrm{C} 1$ (inside the flow) and C3 (outside the flow) and we conclude that there is a pressure balance across the boundary of the plasma outflow region, which is a direct consequence of the boundary layer behavior. As condition for a boundary layer analysis, which is the basis of the reconnection model in Semenov et al. (1983) and Biernat et al. (1987), one assumes $x \gg z$, i.e., elongations normal to the current sheet are small compared to those parallel to it. With $x=3.3 R_{E}$ for TCR 2 and $x=4.7 R_{E}$ for TCR3, the elongation of the outflow region in $x$ is much bigger than in $z$, which is about $1 R_{E}$ (Slavin et al., 2003c). Therefore, one can treat the outflow region still as boundary layer, which is supported by the pressure balance across it.

\section{Conclusions}

We used a series of TCRs during a substorm on 19 September 2001 to estimate the kinetic energy inside the plasma flow regions and the magnetic field energy inside the compression regions above the flow. During this event, five earthward propagating TCRs and one tailward propagating TCR (TCR5) were observed by Cluster. During the second and third TCR, only C3 stayed in the lobe, whereas all other $\mathrm{s} / \mathrm{c}$ were swaped over by the plasma bulge beneath the TCR. Since Eq. (5) is based on integrating bipolar $B_{z}$ signals caused by the TCR, only data from $C 3$ could be used for TCR2 and TCR3. The negative part of the $B_{z}$ variation during the tailward TCR5 is extremely disturbed, and therefore, our method could not be applied for this TCR. Finally, all results are summarized in Tables 2 and 3. It can be seen that the magnetic energy inside the TCR is of the same order as the kinetic energy in the underlying plasma bulge. For the events where all s/c data could be used, the ratio between the magnetic energy inside the TCR and the kinetic energy of the plasma inside the outflow region is very close to the factor of 2, as predicted in Semenov et al. (1998) 
Table 3. Magnetic energy $\Delta W_{B}$ per unit length of the reconnection line inside the investigated TCRs as calculated from Eqs. (4) and (5) for each Cluster spacecraft C1-C4. For TCR2 and TCR3 only C3 observed an TCR, all other spacecraft engulfed the plasma flow region. The same situation appears for TCR5 where C2 was inside the plasma flow. The energy is mainly in the order of $10^{10}$ Joule per unit length of the reconnection line. The last line gives the ratio between the $W_{B}$ and $W_{k}$ from Table 2 of the five earthward TCRs. As can be seen, the ratio between the magnetic energy inside the TCR and the kinetic energy in the underlying plasma bulge is of the same order. For those TCR, where data from all four s/c was available, the value of the ratio is close to the theoretical value of 2, as predicted by Semenov et al. (1998) and Kiehas et al. (2009).

\begin{tabular}{lccccc}
\hline & TCR1 & TCR2 & TCR3 & TCR4 & TCR6 \\
\hline C1 & $5.9 \times 10^{10}$ & n/a & n/a & $3.6 \times 10^{10}$ & $6.6 \times 10^{10}$ \\
C2 & $7.8 \times 10^{10}$ & n/a & n/a & $5.2 \times 10^{10}$ & n/a \\
C3 & $2.4 \times 10^{10}$ & $2.8 \times 10^{10}$ & $7.9 \times 10^{9}$ & $4 \times 10^{10}$ & $5.4 \times 10^{10}$ \\
C4 & $7.4 \times 10^{10}$ & n/a & n/a & $4.5 \times 10^{10}$ & $5.14 \times 10^{10}$ \\
Average C1-C4 & $5.9 \times 10^{10}$ & $2.8 \times 10^{10}$ & $7.9 \times 10^{9}$ & $4.3 \times 10^{10}$ & $5.7 \times 10^{10}$ \\
$\Delta W_{B} / W_{k}$ & 2.4 & 1.1 & 0.1 & 2.4 & 2.4 \\
\hline
\end{tabular}

and Kiehas et al. (2009). It has to be mentioned that the results are given per unit length of the reconnection line due to the 2-D model used in Semenov et al. (1998) and Kiehas et al. (2009). Certainly, this circumstance does not influence the ratio of the magnetic energy inside the TCR to the kinetic plasma energy in the underlying bulge. However, it is shown that TCRs transport a huge amount of magnetic energy (about $10^{10}$ Joule) which is in the order of the kinetic energy inside the outflow region. The transport of magnetic energy inside the Earth's magnetosphere has not been investigated in this form up to now, and the question arises how this amount of energy affects the inner magnetosphere.

Acknowledgements. This work is supported by the Austrian "Fonds zur Förderung der wissenschaftlichen Forschung" under project P20341-N16 and by the Russian Foundation for Basic Research under grant number RFBR 07-05-00776a. Also acknowledged is support by the Austrian Academy of Sciences, "Verwaltungsstelle für Auslandsbeziehungen". S.K. acknowledges financial support from the University Graz in form of a science support sponsorship ("Förderungsstipendium").

Editor in Chief W. Kofman thanks two anonymous referees for their help in evaluating this paper.

\section{References}

Baker, D. N., Pulkkinen, T. I., Angelopoulos, V., Baumjohann, W., and McPherron, R. L.: Neutral line model of substorms: Past results and present view, J. Geophys. Res., 101, 12975-13010, 1996.

Biernat, H. K., Heyn, M. F., and Semenov, V. S.: Unsteady Petschek reconnection, J. Geophys. Res., 92, 3392-3396, 1987.

Borälv, E., Opgenoorth, H. J., Kauristie, K., Lester, M., Bosqued, J.-M., Dewhurst, J. P., Owen, C. J., Dunlop, M., Slavin, J. A., Fazakerley, A., and Perry, C.: Correlation between ground-based observations of substorm signatures and magnetotail dynamics, Ann. Geophys., 23, 997-1011, 2005, http://www.ann-geophys.net/23/997/2005/.
Hones Jr., E. W., Baker, D. N., Bame, S. J., Feldman, W. C., Gosling, J. T., McComas, D. J., Zwickl, R. D., Slavin, J. A., Smith, E. J., and Tsurutani, B. T.: Structure of the magnetotail at 220 earth radii and its response to geomagnetic activity, Geophys. Res. Lett., 11, 5-7, 1984.

Kiehas, S. A., Semenov, V. S., and Biernat, H. K.: Energy budget of the reconnection process, J. Geophys. Res., accepted, 2009.

Meazawa, K.: Magnetotail boundary motion associated with geomagnetic substorms, J. Geophys. Res., 80, 3543-3548, 1975.

Moldwin, M. B. and Hughes, W. J.: Geomagnetic substorm association of plasmoids, J. Geophys. Res., 98, 81-88, 1993.

Moldwin, M. B. and Hughes, W. J.: Observations of earthward and tailward propagating flux rope plasmoids: Expanding the plasmoid model of geomagnetic substorms, J. Geophys. Res., 99, 183-198, 1994.

Moldwin, M. B., Collier, M. R., Slavin, J. A., and Szabo, A.: On the origin of reverse polarity TCRs, Geophys. Res. Lett., 28, 19251928, 2001.

Owen, C. J., Slavin, J. A., Fazakerley, A. N., Dunlop, M. W., and Balogh, A.: Cluster electron observations of the separatrix layer during traveling compression regions, Geophys. Res. Lett., 32, L03104, doi:10.1029/2004GL021767, 2005.

Semenov, V. S., Heyn, M. F., and Kubyshkin, I. V.: Time-dependent Reconnection of the Magnetic Field Lines, Sov. Astron. J., 60, 1138-1147, 1983.

Semenov, V. S., Volkonskaya, N. N., and Biernat, H. K.: Effect of snow plow in bursty magnetic reconnection, Phys. Plasmas, 5, 3242-3248, 1998.

Semenov, V. S., Penz, T., Ivanova, V. V., Sergeev, V. A., Biernat, H. K., Nakamura, R., Heyn, M. F., Kubyshkin, I. V., and Ivanov, I. B.: Reconstruction of the reconnection rate from Cluster measurements: First results, J. Geophys. Res., 110, A11217, doi:10.1029/2005JA011181, 2005.

Sergeev, V. A., Kubyshkina, M. V., Baumjohann, W., Nakamura, R., Amm, O., Pulkkinen, T., Angelopoulos, V., Mende, S. B., Klecker, B., Nagai, T., Sauvaud, J.-A., Slavin, J. A., and Thomsen, M. F.: Transition from substorm growth to substorm expansion phase as observed with a radial configuration of ISTP and Cluster spacecraft, Ann. Geophys., 23, 2183-2198, 2005, 
http://www.ann-geophys.net/23/2183/2005/.

Sharma, A. S., Nakamura, R., Runov, A., Grigorenko, E. E., Hasegawa, H., Hoshino, M., Louarn, P., Owen, C. J., Petrukovich, A., Sauvaud, J.-A., Semenov, V. S., Sergeev, V. A., Slavin, J. A., Sonnerup, B. U. Ö., Zelenyi, L. M., Fruit, G., Haaland, S., Malova, H., and Snekvik, K.: Transient and localized processes in the magnetotail: a review, Ann. Geophys., 26, 9551006, 2008, http://www.ann-geophys.net/26/955/2008/.

Shirai, H., Takada, T. K., Kamide, Y., and Mukai, T.: Enhancements of lobe ion density and velocity associated with plasmoids, J. Geophys. Res., 106, 29935-29942, 2001.

Slavin, J. A., Smith, E. J., Tsurutani, B. T., Sibeck, D. G., Singer, H. J., Baker, D. N., Gosling, J. T., Hones, E. W., and Scarf, F. L.: Substorm associated traveling compression regions in the distant tail - ISEE-3 geotail observations, Geophys. Res. Lett., 11, 657660, 1984.

Slavin, J. A., Lepping, R. P., and Baker, D. N.: IMP-8 observations of traveling compression regions - New evidence for near-earth plasmoids and neutral lines, Geophys. Res. Lett., 17, 913-916, 1990.

Slavin, J. A., Smith, M. F., Mazur, E. L., Baker, D. N., Hones Jr., E. W., Iyemori, T., and Greenstadt, E. W.: ISEE 3 observations of traveling compression regions in the Earth's magnetotail, J. Geophys. Res., 98, 15425-15446, 1993.
Slavin, J. A., Lepping, R. P., Gjerloev, J., Fairfield, D. H., Hesse, M., M., Owen, C. J., Moldwin, M. B., Nagai, T., Ieda, A., and Mukai, T.: Geotail observations of magnetic flux ropes in the plasma sheet, J. Geophys. Res., 108, 1015, doi:10.1029/2002JA009557, 2003a.

Slavin, J. A., Lepping, R. P., Gjerloev, J., Goldstein, M. L., Fairfield, D. H., Acuña, M. H., Balogh, A., Dunlop, M., Kivelson, M. G., Kurana, K., Fazakerley, A., Owen, C. J., Réme, H., and Bosqued, J. M.: Cluster electric current density measurements within a magnetic flux rope in the plasma sheet, Geophys. Res. Lett., 30, 1362, doi:10.1029/2002GL016411, 2003b.

Slavin, J. A., Owen, C. J., Dunlop, M. W., Borälv, E., Moldwin, M. B., Sibeck, D. G., Tanskanen, E., Goldstein, M. L., Fazakerley, A., Balogh, A., Lucek, E., Richter, I., Réme, H., Bosqued, J. M.: Cluster four spacecraft measurements of small traveling compression regions in the near-tail, Geophys. Res. Lett., 30, 2208, doi:10.1029/2003GL018438, 2003c.

Slavin, J. A., Tanskanen, E. I., Hesse, M., Owen, C. J., Dunlop, M. W., Imber, S., Lucek, E. A., Balogh, A., and Glassmeier, K.-H.: Cluster observations of traveling compression regions in the near-tail, J. Geophys. Res., 110, A06207, doi:10.1029/2004JA010878, 2005. 